\title{
Herpes simplex virus 2 encephalitis in a patient heterozygous for a TLR3 mutation
}

Timo Hautala, MD, PhD, Jie Chen, PhD, Laura Tervonen, MD, Terhi Partanen, MD, Satu Winqvist, PhD, Johanna Lehtonen, PhD, Janna Saarela, MD, PhD, Minna Kraatari, MD, Outi Kuismin, MD, PhD, Tytti Vuorinen, MD, PhD, Virpi Glumoff, PhD, Pirjo Åström, PhD, Usko Huuskonen, MD, Lazaro Lorenzo, PhD, Jean-Laurent Casanova, MD, PhD, Shen-Ying Zhang, MD, PhD, and Mikko R.J. Seppänen, MD, PhD
Susceptibility to herpes simplex virus type 1 (HSV-1) encephalitis (HSE-1) in otherwise healthy individuals, in the course of primary infection, can be caused by single-gene inborn errors of Toll-like receptor 3 (TLR3) dependent, interferon (IFN)- $\alpha / \beta$-mediated immunity, ${ }^{1,2}$ or by single-gene inborn errors of snoRNA31. ${ }^{3}$ These variations underlie infections of the forebrain, whereas mutations of DBR1 underlie infections of the brainstem. ${ }^{3} \mathrm{HSV}-2$ encephalitis (HSE-2) is typically observed in neonates, albeit also rarely in older children and adults. ${ }^{4}$ Its manifestations include altered level of consciousness, cranial neuropathies or more extensive brainstem encephalitis, hemiparesis, hemisensory loss, and permanent neurologic deficit. ${ }^{4} \mathrm{MRI}$ in HSE-2 may show normal findings, nonspecific white matter, orbitofrontal, mesial temporal lobe, or brainstem lesions. Inborn errors of immunity underlying HSE-2 have not been described.

A 40-year-old previously healthy woman had suffered from headache and fever of up to $38^{\circ} \mathrm{C}$ for 6 days when she suddenly developed aphasia. At hospital admission, her body temperature was $37.1^{\circ} \mathrm{C}$, blood pressure was $138 / 90 \mathrm{~mm} \mathrm{Hg}$, and vital signs were normal. She was fully conscious and co-operative but aphasic. Her blood hemoglobin level was low $(107 \mathrm{~g} / \mathrm{L}$, normal range 117-155 g/L), with normal C-reactive protein level, blood white cell and thrombocyte counts, and negative blood and urine cultures. Acute phase brain CT, CT angiography and contrast enhanced MRI were unremarkable. CSF showed elevated mononuclear (98\%) white cell count $\left(166 \times 10^{6} / \mathrm{L}\right.$; normal $\left.<3 \times 10^{6} / \mathrm{L}\right)$ and high protein concentration $(1,192 \mathrm{mg} / \mathrm{L}$; normal range $150-500 \mathrm{mg} / \mathrm{L}$ ). CSF was positive for HSV-2 and negative for HSV-1 nucleic acid by automated and accredited real-time PCR (artus HSV-1/2 PCR Kits; QIAsymphony SP, Rotor-Gene Q, Qiagen, Hilden, Germany). EEG showed left focal frontotemporobasal $2 \mathrm{~Hz}$ slow wave activity consistent with viral encephalitis (figure). She received IV acyclovir for 21 days with improvement in aphasia. Neuropsychological assessment 1 month later revealed poor word fluency, problems with memory interference, and delayed word list memory. The patient continued to suffer from lassitude and mild depression at least for over 12 months after the acute episode. Her presentation was consistent with International the Encephalitis Consortium diagnostic criteria for encephalitis. ${ }^{5}$ After the acute episode, she developed frequently recurrent eczema herpeticum of lower back tested positive for HSV-2 nucleic acid, suggesting recent primary HSV-2 infection. She did not suffer from genital herpes. This was controlled with peroral prophylactic valacyclovir $500 \mathrm{mg}$ twice daily.

\author{
Correspondence \\ Dr. Hautala \\ timo.hautala@oulu.fi
}




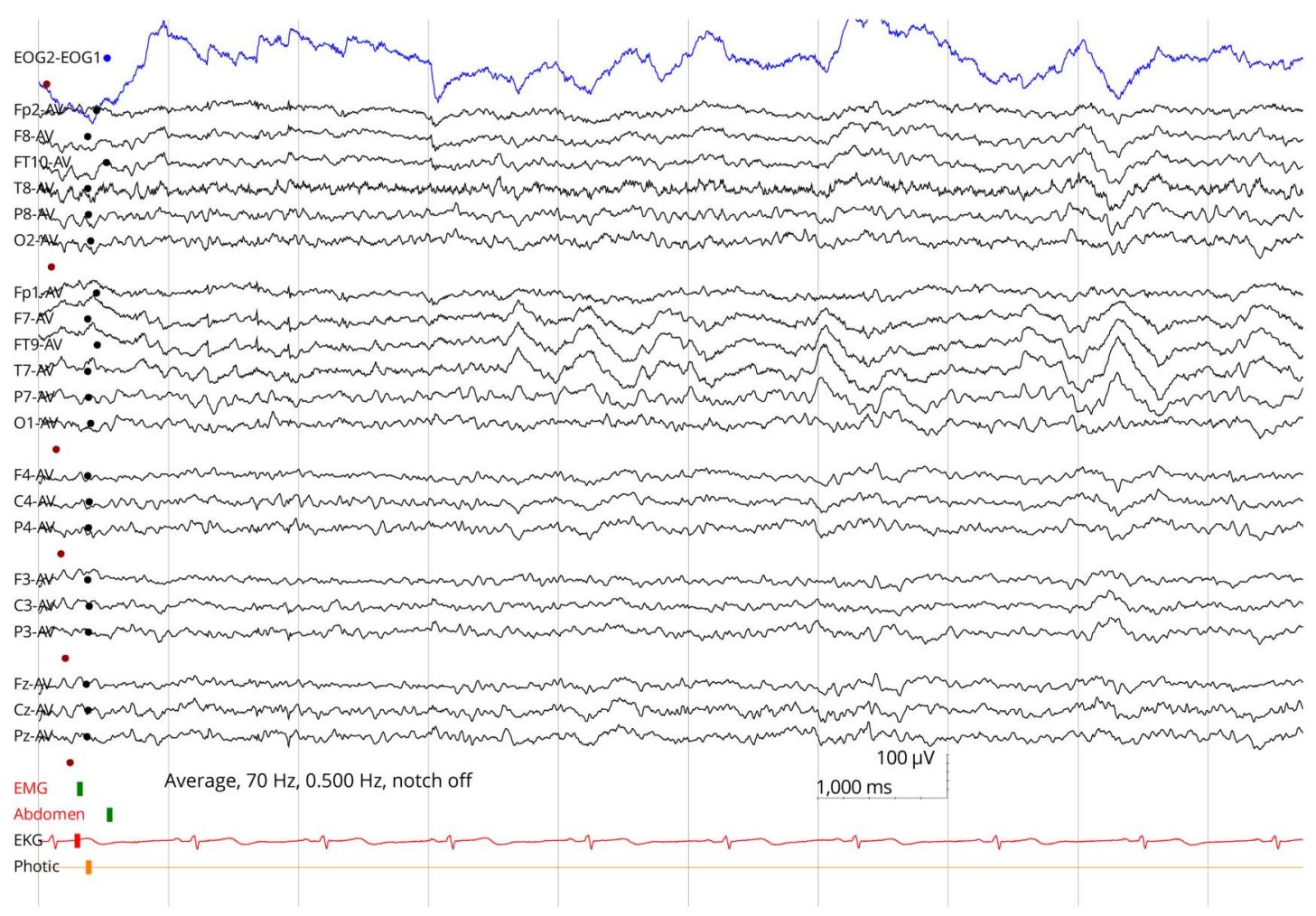

Normal background activity and the patient is awake.

The patient was remitted for immunologic evaluation. Family history was unremarkable; she had no history of general infection susceptibility, autoimmunity, or secondary immunodeficiency. Blood white cell and lymphocyte $\left(\mathrm{CD} 19^{+} \mathrm{B}, \mathrm{CD} 4^{+}\right.$ and $\left.\mathrm{CD}^{+} \mathrm{T}, \mathrm{CD} 16^{+} \mathrm{CD} 56^{+} \mathrm{NK}\right)$ counts and percentages of $\mathrm{T}$ and $\mathrm{B}$ lymphocyte subclasses were normal, suggesting normal maturation. She was positive for anti-HSV2 immunoglobulin G (IgG) and negative for anti-HSV1 IgG. Whole exome sequencing showed a heterozygous rs147431766 TLR3 (chr4: 187005064C>T, ENSG00000164342:ENST00000296795: exon4:c.C2224T:p.L742F) variant, enriched over 23-fold in the Finnish compared with non-Finnish Europeans (allele frequency 0.01621 vs 0.0006819 , Genome Aggregation Database; gnomad.broadinstitute.org/). TLR3 p.L742F variant is in vitro severely hypomorphic based on experiments on TLR3deficient P2.1 fibrosarcoma cell line, and the production of IFN- $\lambda$ and interleukin- 6 in response to TLR3 activation by poly(I:C) stimulation was impaired in previously tested SV40 immortalized human skin fibroblasts heterozygous for this variation. ${ }^{6}$ She was also found to be positive for TRF1interacting nuclear factor 2 (TINF2) variant p.Y312* (rs201677741) previously associated with Ewing sarcoma. Pathogenic TINF variants are associated with autosomal dominant dyskeratosis congenita and pathologic telomere lengths (OMIM 604319). However, her telomere length was within the normal interval compared with healthy controls of similar age. Patient's father had died of aggressive cholangiocarcinoma, and her mother has had one episode of varicella zoster skin infection. TLR3 p.L742F and TINF2 p.Y312* variants were inherited from the mother. Patient's sister had suffered from recurrent laboratory confirmed cutaneous HSV2 infections. Her targeted testing did not reveal TLR3 p.L742F or TINF2 p.Y312* variants.

Previous evidence shows that inborn errors in TLR3-mediated immune response may explain HSE-1 in a subset of patients. ${ }^{1-3}$ Our patient suffered an HSE-2 episode associated with impaired TLR3-mediated antiviral response. To our knowledge, this is the first report of a genetic mechanism potentially explaining HSE-2 in immunocompetent patients. The TLR3 p.L742F variant found in our patient is enriched in Finnish population; we can only speculate that this property may associate with HSV-2 CNS infections in Finland. ${ }^{7}$ We emphasize that our report does not prove causality between TLR3 deficiency and HSE-2 but suggests that TLR3 signaling defects may need to be contemplated in otherwise healthy HSE-2 patients.

\section{Study funding}

No targeted funding reported.

\section{Disclosure}

The authors report no disclosures. Go to Neurology.org/NG for full disclosures. 


\section{Publication history}

Received by Neurology: Genetics August 13, 2020. Accepted in final form September 22, 2020.

Appendix Authors

\begin{tabular}{|c|c|c|}
\hline Name & Location & Contribution \\
\hline $\begin{array}{l}\text { Timo Hautala, MD, } \\
\text { PhD }\end{array}$ & $\begin{array}{l}\text { University of Oulu, } \\
\text { Finland }\end{array}$ & $\begin{array}{l}\text { Patient care and study } \\
\text { design }\end{array}$ \\
\hline Jie Chen, PhD & $\begin{array}{l}\text { Rockefeller } \\
\text { University, } \\
\text { New York, NY }\end{array}$ & Laboratory analysis \\
\hline $\begin{array}{l}\text { Laura Tervonen, } \\
\text { MD }\end{array}$ & $\begin{array}{l}\text { Oulu University } \\
\text { Hospital, Finland }\end{array}$ & $\begin{array}{l}\text { Patient care and } \\
\text { diagnostics }\end{array}$ \\
\hline Terhi Partanen, MD & $\begin{array}{l}\text { Oulu University } \\
\text { Hospital, Finland }\end{array}$ & $\begin{array}{l}\text { Study design and } \\
\text { analysis }\end{array}$ \\
\hline Satu Winqvist, PhD & $\begin{array}{l}\text { Oulu University } \\
\text { Hospital, Finland }\end{array}$ & $\begin{array}{l}\text { Neuropsychological } \\
\text { evaluation }\end{array}$ \\
\hline $\begin{array}{l}\text { Johanna Lehtonen, } \\
\text { PhD }\end{array}$ & $\begin{array}{l}\text { University of Helsinki, } \\
\text { Finland }\end{array}$ & Laboratory analysis \\
\hline $\begin{array}{l}\text { Janna Saarela, MD, } \\
\text { PhD }\end{array}$ & $\begin{array}{l}\text { University of Helsinki, } \\
\text { Finland }\end{array}$ & $\begin{array}{l}\text { Major role in data } \\
\text { analysis }\end{array}$ \\
\hline Minna Kraatari, MD & $\begin{array}{l}\text { Oulu University } \\
\text { Hospital, Finland }\end{array}$ & Genetic evaluation \\
\hline $\begin{array}{l}\text { Outi Kuismin, MD, } \\
\text { PhD }\end{array}$ & $\begin{array}{l}\text { Oulu University } \\
\text { Hospital, Finland }\end{array}$ & Genetic evaluation \\
\hline $\begin{array}{l}\text { Tytti Vuorinen, MD, } \\
\text { PhD }\end{array}$ & $\begin{array}{l}\text { University of Turku, } \\
\text { Finland }\end{array}$ & Virologic evaluation \\
\hline Virpi Glumoff, PhD & $\begin{array}{l}\text { University of Oulu, } \\
\text { Finland }\end{array}$ & Immunologic evaluation \\
\hline
\end{tabular}

Appendix (continued)

\begin{tabular}{lll}
\hline Name & Location & Contribution \\
\hline Pirjo Åström, PhD & $\begin{array}{l}\text { University of Oulu, } \\
\text { Finland }\end{array}$ & Laboratory analysis \\
\hline $\begin{array}{l}\text { Usko Huuskonen, } \\
\text { MD }\end{array}$ & $\begin{array}{l}\text { Oulu University } \\
\text { Hospital, Finland }\end{array}$ & EEG analysis \\
\hline $\begin{array}{l}\text { Lazaro Lorenzo, } \\
\text { PhD }\end{array}$ & $\begin{array}{l}\text { Paris Descartes } \\
\text { University, France }\end{array}$ & Immunologic analysis \\
\hline $\begin{array}{l}\text { Jean-Laurent } \\
\text { Casanova, MD, PhD }\end{array}$ & $\begin{array}{l}\text { Rockefeller } \\
\text { University, } \\
\text { New York, NY }\end{array}$ & $\begin{array}{l}\text { Study design and } \\
\text { manuscript preparation }\end{array}$ \\
\hline $\begin{array}{l}\text { Shen-Ying Zhang, } \\
\text { MD, PhD }\end{array}$ & $\begin{array}{l}\text { Rockefeller } \\
\text { University, } \\
\text { New York, NY }\end{array}$ & $\begin{array}{l}\text { Study design and } \\
\text { manuscript preparation }\end{array}$ \\
\hline $\begin{array}{l}\text { Mikko R.J. } \\
\text { Seppänen, MD, PhD }\end{array}$ & $\begin{array}{l}\text { Helsinki University } \\
\text { Hospital, Finland }\end{array}$ & $\begin{array}{l}\text { Study design and } \\
\text { manuscript preparation }\end{array}$ \\
\hline
\end{tabular}

\section{References}

1. Zhang SY, Jouanguy E, Ugolini S, et al. TLR3 deficiency in patients with herpes simplex encephalitis. Science 2007;317:1522-1527.

2. Lim HK, Seppänen M, Hautala $T$, et al. TLR3 deficiency in herpes simplex encephalitis: high allelic heterogeneity and recurrence risk. Neurology 2014;83: $1888-1897$.

3. Zhang SY. Herpes simplex virus encephalitis of childhood: inborn errors of central nervous system cell-intrinsic immunity. Hum Genet 2020;139:911-918.

4. Berger JR, Houff S. Neurological complications of herpes simplex virus type 2 infection. Arch Neurol 2008;65:596-600.

5. Venkatesan A, Tunkel AR, Bloch KC, et al. Case definitions, diagnostic algorithms, and priorities in encephalitis: consensus statement of the international encephalitis consortium. Clin Infect Dis 2013;57:1114-1128.

6. Partanen T, Chen J, Lehtonen J, et al. Heterozygous TLR3 mutation in patients with hantavirus encephalitis. J Clin Immunol 2020;40:1156-1162.

7. Kupila L, Vuorinen T, Vainionpää R, Hukkanen V, Marttila RJ, Kotilainen P. Etiology of aseptic meningitis and encephalitis in an adult population. Neurology 2006;66: $75-80$ 


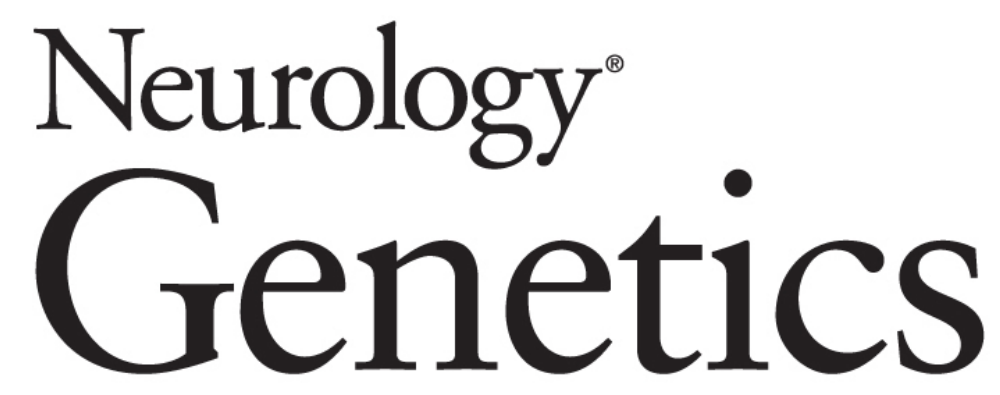

\section{Herpes simplex virus 2 encephalitis in a patient heterozygous for a TLR3 mutation}

Timo Hautala, Jie Chen, Laura Tervonen, et al.

Neurol Genet 2020;6;

DOI 10.1212/NXG.0000000000000532

This information is current as of November 25, 2020

Updated Information \&

Services

References

Subspecialty Collections

Permissions \& Licensing

Reprints including high resolution figures, can be found at: http://ng.neurology.org/content/6/6/e532.full.html

This article cites 7 articles, 1 of which you can access for free at: http://ng.neurology.org/content/6/6/e532.full.html\#\#ref-list-1

This article, along with others on similar topics, appears in the following collection(s):

All Genetics

http://ng.neurology.org//cgi/collection/all_genetics

All Immunology

http://ng.neurology.org//cgi/collection/all_immunology

Encephalitis

http://ng.neurology.org//cgi/collection/encephalitis

Viral infections

http://ng.neurology.org//cgi/collection/viral_infections

Information about reproducing this article in parts (figures,tables) or in its entirety can be found online at:

http://ng.neurology.org/misc/about.xhtml\#permissions

Information about ordering reprints can be found online:

http://ng.neurology.org/misc/addir.xhtml\#reprintsus

Neurol Genet is an official journal of the American Academy of Neurology. Published since April 2015, it is an open-access, online-only, continuous publication journal. Copyright Copyright @ 2020 The Author(s). Published by Wolters Kluwer Health, Inc. on behalf of the American Academy of Neurology.. All rights reserved. Online ISSN: 2376-7839.

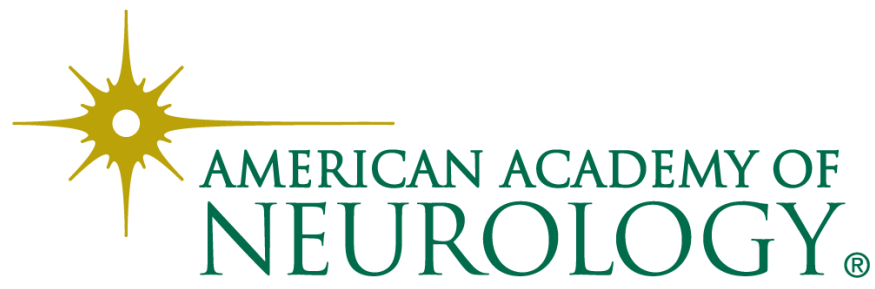

